\title{
Sequence requirements for micro RNA processing and function in human cells
}

\author{
YAN ZENG ${ }^{\mathbf{1}}$ and BRYAN R. CULLEN ${ }^{1,2}$ \\ ${ }^{1}$ Howard Hughes Medical Institute, ${ }^{2}$ Department of Molecular Genetics and Microbiology, Duke University Medical Center, \\ Durham, North Carolina 27710, USA
}

\begin{abstract}
Most eukaryotes encode a substantial number of small noncoding RNAs termed micro RNAs (miRNAs). Previously, we have demonstrated that miR-30, a 22-nucleotide human miRNA, can be processed from a longer transcript bearing the proposed miR-30 stem-loop precursor and can translationally inhibit an mRNA-bearing artificial target sites. We also demonstrated that the miR-30 precursor stem can be substituted with a heterologous stem, which can be processed to yield novel miRNAs and can block the expression of endogenous mRNAs. Here, we show that a second human miRNA, termed miR-21, can also be effectively expressed when its precursor forms part of a longer mRNA. For both miR-30 and miR-21, mature miRNA production was highly dependent on the integrity of the precursor RNA stem, although the underlying sequence had little effect. In contrast, the sequence of the terminal loop affected miRNA production only moderately. Processing of the initial, miR-30-containing transcript led to the production of not only mature miR-30 but also to the largely nuclear excision of an $\sim 65$-nucleotide RNA that is likely to represent an important intermediate in miR-30 processing. Consistent with this hypothesis, mutations that affected mature miR-30 production inhibited expression of this miR-30 pre-miRNA to an equivalent degree. Although point mutations could block the ability of both miR-30 and miR-21 to inhibit the translation of mRNAs bearing multiple artificial miRNA target sites, single point mutations only attenuated the miRNA-mediated inhibition of genes bearing single, fully complementary targets. These results suggest that miRNAs, and the closely similar small interfering RNAs, cannot totally discriminate between RNA targets differing by a single nucleotide.
\end{abstract}

Keywords: RNA structure; RNA interference (RNAi); small interfering RNAs (siRNAs); translation

\section{INTRODUCTION}

A family of $\sim 22$-nucleotide (nt) noncoding RNAs termed microRNAs (miRNAs) has been identified in eukaryotic organisms ranging from nematodes to humans (LagosQuintana et al. 2001; Lau et al. 2001; Lee and Ambros 2001). The founding members of this family of small, noncoding RNAs are the Caenorhabditis elegans lin-4 and let-7 miRNAs (Lee et al. 1993; Reinhart et al. 2000). Lin-4 and let-7 are also called small temporal RNAs because their mutational inactivation affects developmental timing (Banerjee and Slack 2002). These two miRNAs exert their functions by inhibiting the translation of target mRNAs that contain $3^{\prime}$ untranslated region (3'UTR) sequences that are partially complementary (Lee et al. 1993; Reinhart et al. 2000). The mechanism underlying this inhibition is un-

Reprint requests to: Bryan R. Cullen, Howard Hughes Medical Institute, Duke University Medical Center, Box 3025, Durham, NC 27710, USA; e-mail: culle002@mc.duke.edu.

Article and publication are at http://www.rnajournal.org/cgi/doi/ 10.1261/rna.2780503. known; in the cases that have been examined, lin-4 does not significantly affect the expression level of target mRNAs (Olsen and Ambros 1999). Lin-4 and let-7 are predicted to occupy one side of an imperfectly base-paired stem in a 70-nt RNA hairpin encoded within the C. elegans genome (Lee et al. 1993; Reinhart et al. 2000). The expression of these longer, precursor RNAs has been detected, although it seems unlikely that this $\sim 70$-nt precursor defines the actual $5^{\prime}$ and $3^{\prime}$ ends of the primary transcript that eventually give rise to the mature miRNA. Recently, more than one hundred new miRNAs have been identified in nematodes, fruitflies, mice, and humans (Lagos-Quintana et al. 2001, 2002; Lau et al. 2001; Lee and Ambros 2001; Mourelatos et al. 2002). Some miRNAs are expressed in a developmental stage-specific or tissue-specific manner, whereas others appear to be constitutively present. Like lin-4 and let-7, these miRNAs are also encoded within one arm of a putative 70-nt RNA stem-loop precursor. None of these new miRNAs has any ascribed function as yet.

There are similarities and differences between miRNAs and another class of RNAs called small interfering RNAs 
(siRNAs) (for review, see Hutvágner and Zamore 2002). The siRNAs are normally processed from long doublestranded (ds) RNAs during RNA interference (RNAi) in animals or during post-transcriptional gene silencing (PTGS) in plants (Hamilton and Baulcombe 1999; Hammond et al. 2000; Zamore et al. 2000). RNAi and PTGS induce the selective degradation of mRNAs that are identical or highly homologous in sequence to the dsRNA inducer, and the siRNA is the guide RNA that directs this degradation. Dicer, a conserved RNase III family ribonuclease, is required for the production of both siRNAs and miRNAs (Bernstein et al. 2001; Grishok et al. 2001; Hutvágner et al. 2001; Knight and Bass 2001; Lee and Ambros 2001). Both siRNAs and miRNAs are $\sim 22 \mathrm{nt}$ in length, although miRNAs are usually single-stranded, whereas siRNAs are believed to be predominantly double-stranded, with $2 \mathrm{nt} 3^{\prime}$ overhangs formed by dicer cleavage. The incorporation of an siRNA into the RNA-induced silencing complex (RISC), a large ribonucleoprotein complex, generates a specific endonuclease that cleaves target mRNAs (Hammond et al. 2000). In contrast, in the case of lin-4 miRNA, target mRNAs are not degraded (Olsen and Ambros 1999). The predominant constitutive $\sim 22$-nt cellular RNA species are miRNAs, whereas siRNAs are normally induced after viral infection or when dsRNAs are introduced into cells artificially, although active transposons also appear to lead to siRNA production (Hutvágner and Zamore 2002). Thus, RNAi-related mechanisms are primed for suppressing transposon activity and viral infection, whereas miRNAs minimally have roles in development. Nevertheless, there may be significant overlap between cellular pathways using siRNAs and miRNAs that remain to be determined.

We are interested in miRNA biogenesis, their functions, and their links to the RNAi pathway. Previously, we showed that the human miR-30 stem-loop RNA precursor, when placed under the control of the RNA polymerase II-dependent cytomegalovirus immediate early (CMV-IE) promoter, would generate correctly processed miR-30 miRNA in transfected human cells (Zeng et al. 2002). The miR-30 miRNA inhibited the translation of a reporter gene bearing four miR-30 target sites in its $3^{\prime}$ UTR, just like lin-4 in $C$. elegans. We also showed that the miR-30 miRNA precursor could be exploited as a carrier to express novel miRNAs/ siRNAs and that these novel miRNAs/siRNAs could effectively block the expression of endogenous genes by inducing mRNA degradation. Here, we have used this system to further define the sequence requirements for miRNA processing and function in human cells. We found that although miRNA precursors are tolerant of mutations in the body of the stem and, to a lesser extent, the terminal loop, disrupting the base-pairing at the base of the stem has a marked deleterious effect on miRNA processing. Single point mutations in the miR-30 or miR-21 miRNA were identified that render these miRNAs inactive in inhibiting the trans- lation of indicator mRNAs bearing multiple artificial target sites. In contrast, single nucleotide mismatches between a designed miRNA/siRNA and an otherwise perfectly complementary mRNA target site reduced, but did not abolish, inhibition of target mRNA expression.

\section{RESULTS}

\section{Mutagenesis of the miR-30 miRNA precursor}

We constructed a panel of mutants of the proposed miR-30 RNA precursor (Table 1), expressed them under the control of the CMV-IE promoter as described previously (Fig. 1A; Zeng et al. 2002), and analyzed their expression in transfected $293 \mathrm{~T}$ cells by Northern blotting and primer extension, which gave comparable results in terms of detecting mature miRNA (Fig. 1). Most mutants were still able to produce mature miR-30, although some at a lower level than wild-type miR-30. For example, the miR-30( $\Delta$ AGAUG) mutation, which shortens the predicted terminal loop to 4 nt (and the precursor in the process), gave a low but still detectable level of miR-30 expression (Table 1; Fig. 1B, lane 3; Fig. 1C, lane 3). In contrast, changing the sequence of the terminal loop, or shortening it to $11 \mathrm{nt}$, did not have any noticeable effect [Table 1; mutants miR-30(UGAGA), miR30(GCG), and miR-30(ACAGCG)]. Although eliminating the bulges in the predicted miR-30 stem was of little consequence [mutants miR-30( $\Delta \mathrm{UC})$ and $\operatorname{miR}-30(\mathrm{G})]$, creating a larger bulge was clearly detrimental to miR-30 production [Table 1; mutant miR-30(UC)]. Changing the stem sequence without perturbing base-pairing was neutral [e.g., miR-30(GA/UC), miR-30(A), and mir30(C); Fig. 1B, lanes $4,5]$. We identified one mutant, miR-30(GAG), that essentially knocked out miR-30 expression (Fig. 1B, lane 6; Fig. 1C, lane 4). This mutant disrupts base-pairing at the base of the predicted stem (Table 1). When the base-pairing, but not the sequence, was restored in miR-30(CUC/GAG), miR-30 expression was also restored (Fig. 1B, lane 7; Fig. 1C, lane 5).

Using Northern blots we also detected a transcript of $\sim 65$ nt (indicated by an arrowhead in Fig. 1C) that likely represents an intermediate in miR-30 processing. A very recent paper by Lee et al. (2002) demonstrates that miR-30 processing proceeds by an $\sim 65$-nt intermediate, termed a premiRNA, that coincides with the apical $65 \mathrm{nt}$ of the proposed miR-30 precursor RNA stem-loop structure shown in Table 1. Using RNAse protection analysis (RPA), we were able to confirm this report and were further able to map the $5^{\prime}$ end of the $\sim 65$-nt miR-30 pre-miRNA intermediate to the most $5^{\prime} \mathrm{U}$ residue in the predicted miR-30 precursor RNA stemloop (Table 1) (data not shown). Interestingly, this $U$ residue also forms the $5^{\prime}$ end of the anti-miR-30 miRNA that is excised from the $5^{\prime}$ arm of the miR-30 miRNA precursor. The miR-30 precursor is unusual in giving rise to miRNAs derived from both the $5^{\prime}$ and $3^{\prime}$ arm of the precursor stem, 
TABLE 1. Selected miR-30 mutants

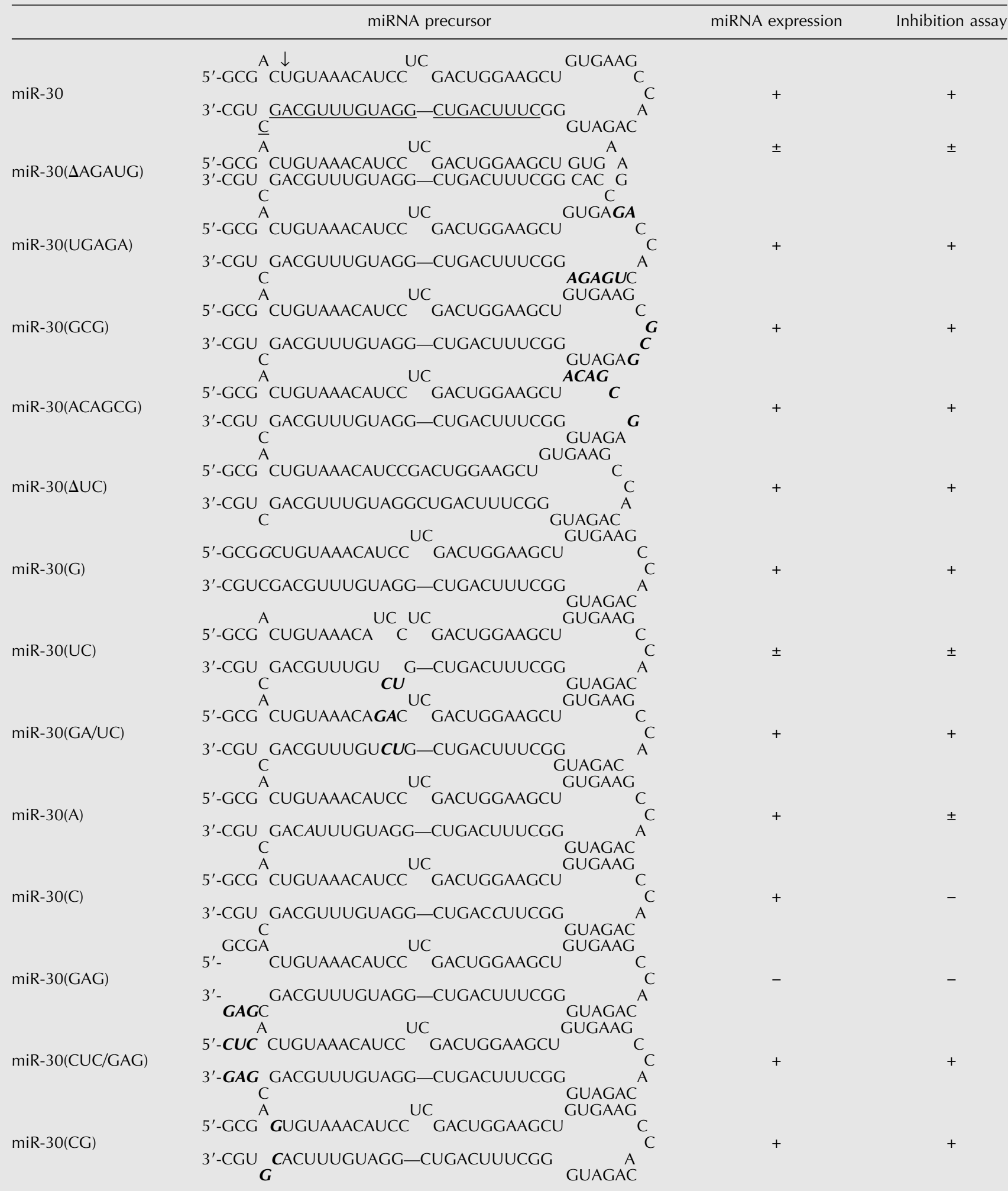

The mature miR-30 sequence is underlined. Mutations are shown in bold and italic fonts. The arrow shown at the top of the wild-type miR-30 sequence indicates the $5^{\prime}$ end $( \pm 1 \mathrm{nt}$ ) of the miR-30 pre-miRNA intermediate (Fig. 1 ) as determined by RPA. Plasmids encoding the wild-type and mutants precursors were transfected into $293 \mathrm{~T}$ cells, tested for mature miRNA expression by primer extension, and for their ability to inhibit the expression of indicator constructs encoding the CAT or luciferase gene linked to four miR-30 target sites.,$+ 50-100 \%$ of wild-type levels;,$\pm<50 \% ;-, \sim 0 \%$. 
$\mathbf{A}$

pCMV-miR-30:

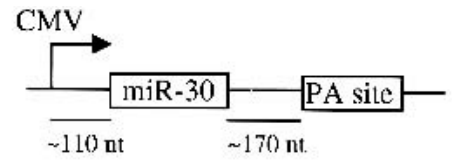

B

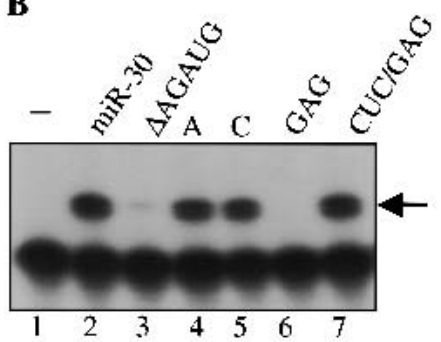

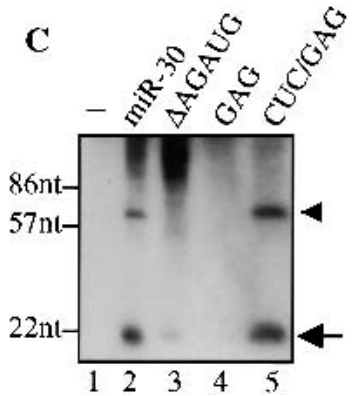

FIGURE 1. Analysis of miR-30 expression level. (A) A schematic of pCMV-miR-30. The predicted miR-30 precursor (Table 1) is flanked by $X h o I$ sites, and the basal stem is, therefore, predicted to be extended by $6 \mathrm{bp}$. PA site, polyadenylation site. (B) Primer extension assay to detect miR-30 in transfected 293T cells. Lane 1, RNA from cells transfected with pBC12/CMV; lane 2, pCMV-miR-30; lanes 3-7, various miR-30 mutants. (C) Northern analysis of miR-30 expression. Lane 1, pBC12/CMV; lane 2, pCMV-miR-30; lanes 3-5, various miR-30 mutants. The arrow indicates the predicted position of the mature miR-30 and the arrowhead indicates the $\sim 65$-nt mir-30 pre-miRNA on the Northern blot. Positions of known DNA markers are shown at left.

whereas other miRNA precursors normally generate only a single stable mRNA (Lagos-Quintana et al. 2002). As expected, for all the miR-30 precursor mutants analyzed, the pattern of anti-miR-30 expression in transfected cells was closely correlated with miR-30 expression (data not shown). Importantly, mutations that inhibited production of the mature 22 -nt miR-30 (and anti-miR-30) miRNA also inhibited production of the pre-miRNA intermediate (Fig. 1C). This observation suggests that mutations that inhibited mature miR-30 production were actually acting primarily by blocking production of the pre-miRNA intermediate.

We then tested how these mutations affected the ability of miR-30 to specifically inhibit the expression of an indicator chloramphenicol acetyl transferase (CAT) mRNA. The indicator plasmid used contains four copies of a miR30 target site, as described previously (Zeng et al. 2002). Each target site was designed so that miR-30 is predicted to form two RNA helices ( 9 or 10 bp long) with the target separated by a bulge in the middle (Fig. 2A), analogous to known target sites for $C$. elegans lin-4 and let-7 (Banerjee and Slack 2002). There are three categories of miRNA precursor mutants. The first category includes mutations that severely reduced or eliminated miR-30 expression (such as the $\triangle$ AGAUG and GAG mutants). As expected, these mutants were similarly defective in the inhibition assay (Fig. 2B). The second category consists of mutations that had no significant effect on miR-30 production, and were also not predicted to affect base-pairing between miR-30 and the target sites. These mutants were essentially as active as wild- type miR-30 in inhibiting CAT expression [Table 1, mutants miR-30(UGAGA), miR-30(GCG), miR-30(ACAGCG), miR-30(G), miR-30(GA/UC), and miR-30( $\Delta \mathrm{UC})]$. The final category includes mutants that are predicted to affect the miR-30:target mRNA interaction. We found that mutations predicted to reduce RNA helix formation toward the center [miR-30(A) and miR-30(C); Fig. 2] were far more deleterious than a mutation predicted to affect base-pairing at the end of a helix [Fig. 2; mutant miR-30(CG)]. Although these results are generally predictable, there may be some subtleties in the contribution of individual positions that deserve future mechanistic studies, for example, the miR$30(\mathrm{C})$ mutant failed to repress CAT expression whereas the similar miR-30(A) mutant retained partial activity (Fig. 2B).

\section{Mutagenesis of the miR-21 miRNA precursor}

To determine whether our system was applicable to other miRNAs, we substituted the full-length, predicted miRNA precursors encoding four other miRNAs (human let-7,

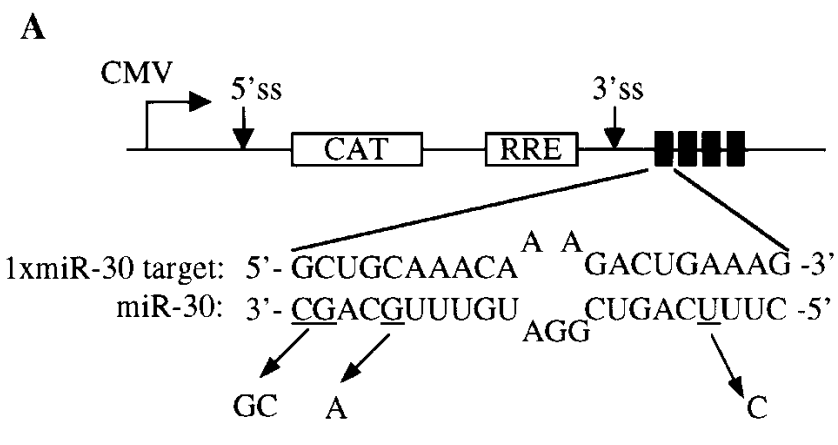

B

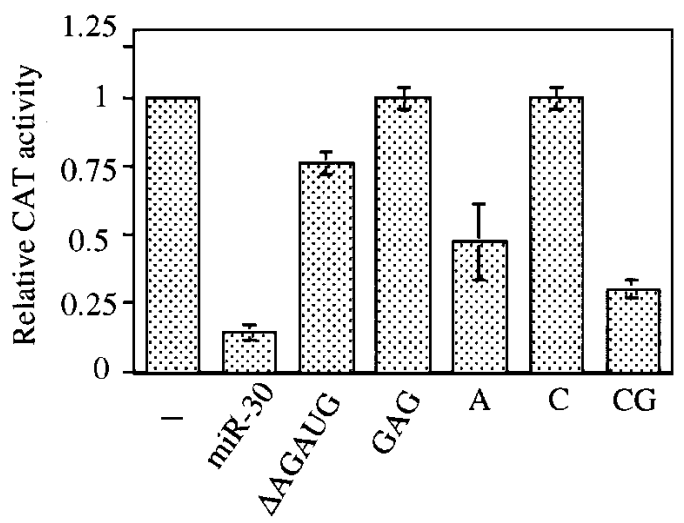

FIGURE 2. Biological activity of miR-30 mutants. (A) Schematic of the indicator construct with four copies of a miR-30 target site (black boxes). The predicted base-pairing between miR-30 and the mRNA target, and the position of three miR-30 mutations are shown. (B) CAT expression in 293T cells cotransfected with the indicator plasmid pcRev and various miR-30 expression plasmids, as well as with the $\mathrm{pBC} 12 / \mathrm{CMV} / \beta$-gal internal control plasmid. CAT activities were normalized using the $\beta$-gal activities, and set at 1 for cells transfected with the $\mathrm{pBC} 12 / \mathrm{CMV}$ negative control plasmid (marked as -). Error bars represent standard deviations. 
miR-21, miR-27, and C. elegans lin-4) (Lagos-Quintana et al. 2001) in place of the miR-30 precursor in the same CMV-IE-based expression plasmid (Fig. 1A), transfected 293T cells, and examined miRNA expression by primer extension. miR-21 was the only miRNA that was detected significantly over endogenous levels (Fig. 3A, lane 2). Unlike the case with miR-30, anti-miR-21 was not detected (Fig. 3A, lanes 10-12).

We introduced mutations into the miR-21 precursor at positions analogous to those in miR-30 (Table 2). There is a general correlation between miR-21 mutant phenotypes and miR-30 mutant phenotypes. For instance, the miR21(GGU) mutation, which opens the base of the predicted precursor stem, practically eliminated expression of mature
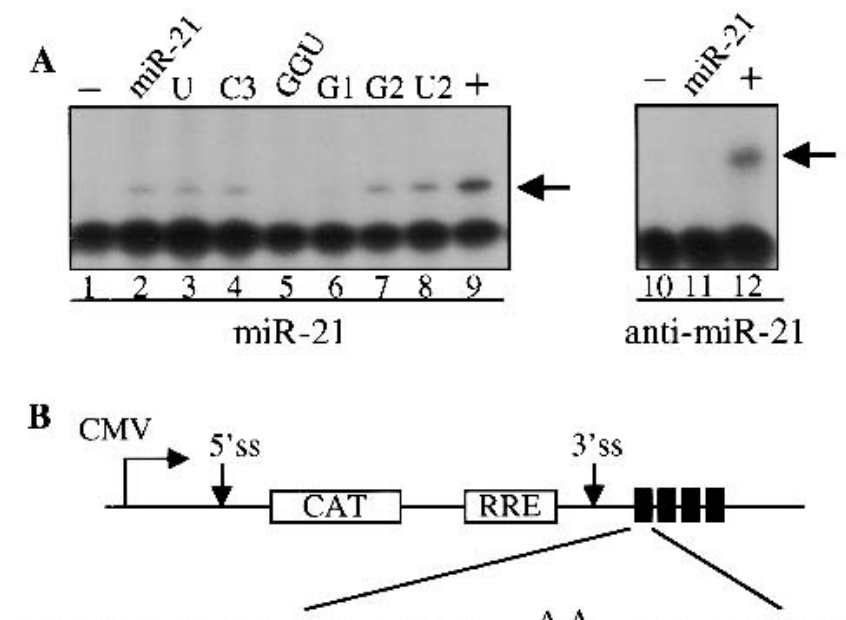

Ix miR-2 1 target: 5' - UCAACAUCAG ${ }^{\text {A A }}$ GAUAAGCUA -3, miR-21: 3'- AGUUGU $\underline{\text { AGUC }}$ AGA $_{\text {CUAUU }}$ CGAL- 5

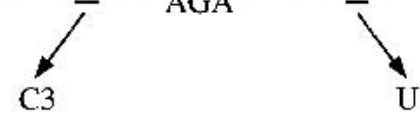

C

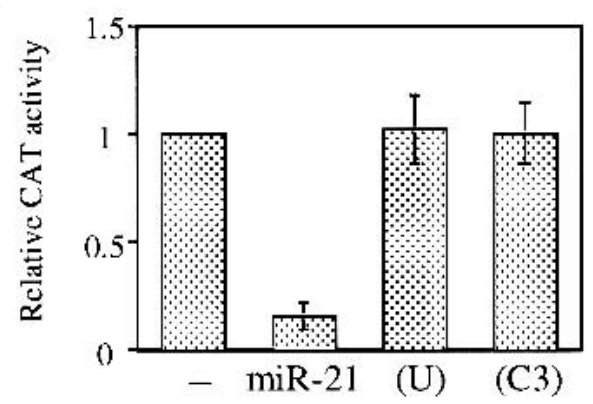

FIGURE 3. Analysis of miR-21 mutants. (A) Primer extension to detect mature miR-21 (lanes 1-9) or anti-miR-21 (lanes 10-12) in transfected cells. -, Cells transfected with pBC12/CMV (lane 1); +, 20 pg of a DNA oligonucleotide encoding mature miR-21 (lane 9) or the predicted anti-miR-21 (lane 12). Arrows indicate the expected position of the mature miRNA. (B) Schematic of the CAT indicator plasmid containing four miR-21 target sites (black boxes). The predicted partial base-pairing between miR-21 and its artificially designed target site, and the positions of two miR-21 point mutations, miR-21(C3) and miR-21(U), are shown. $(C)$ CAT assay. See Figure 2B legend for details.
miR-21 (Fig. 3A, lane 5), just like the equivalent miR30(GAG) mutation (Table 1). Base-pairing at the G1 position appeared to be more important than the other two positions (G2 and U2), as the miR-21(G1) mutant expressed the lowest amount of miR-21 (Fig. 3A, cf. lane 6 and lanes 7,8). Interestingly, we could not identify such a critical position in the miR-30 precursor (data not shown).

We then asked whether miR-21 shared with miR-30 the ability to inhibit gene expression. A miR-21 target site was designed in the same way as the miR-30 target, and four copies of the miR-21 target sites were used to replace the four miR-30 sites in the CAT indicator construct (Fig. 3B). Coexpressing miR-21 with the indicator mRNA specifically reduced CAT activity by $\sim 85 \%$ (Fig. $3 \mathrm{C}$ ). Two point mutations, miR-21(U) and miR-21(C3), designed to disrupt base-pairing near the center of the helix, blocked the ability of miR-21 to repress indictor gene expression (Fig. 3C), although the corresponding miRNAs were expressed at a similar level as miR-21 (Fig. 3A, cf. lanes 2-4).

\section{Inhibition of luciferase indicator gene expression by native miRNAs}

To further test the generality of the inhibition of gene expression by miRNAs, we generated an indicator plasmid expressing the firefly luciferase gene and placed four copies of either the miR-30 or miR-21 target site in the $3^{\prime}$ UTR (Fig. 4A). We cotransfected miR-30 or miR-21 expression plasmids along with the firefly luciferase indicator, a Renilla luciferase expression plasmid and a $\beta$-gal expression plasmid as internal controls, into $293 \mathrm{~T}$ cells, and performed dual-luciferase assays $2 \mathrm{~d}$ later. As expected, the miR-30 expression plasmid specifically repressed luciferase activity from the indicator bearing four copies of the miR-30 target, but not from the indicator plasmid bearing four miR-21 sites (Fig. 4B). In contrast, the miR-21 expression plasmid gave the opposite result, and neither miR-30 nor miR-21 repressed a luciferase indicator plasmid lacking inserted target sites (Fig. 4; data not shown). Mutations of the miR-30 or miR-21 miRNA precursor affected luciferase expression the same way as they did with CAT expression (Fig. 4; not shown). Neither miR-30 nor miR-21 appreciably reduced the steady-state levels of the target luc mRNA (Fig. 4D,E, lanes 2-4). This result is consistent with previous data suggesting that miRNAs can repress the translation of mRNAs bearing multiple, partially mismatched target sites (Olsen and Ambros 1999; Zeng et al. 2002).

\section{RNA target specificities of plasmid-encoding siRNAs}

Previously, we showed that by substituting the stem sequences in the miR-30 precursor with designed base-pairing sequences, novel $\sim 22$-nt miRNAs could be generated that appeared to act as siRNAs to initiate RNAi (Zeng et al. 2002). That is, these novel miRNAs/siRNAs inhibited the 
TABLE 2. Selected miR-21 mutants

\begin{tabular}{|c|c|c|c|}
\hline & miRNA precursor & miRNA expression & Inhibition assay \\
\hline miR-21 & 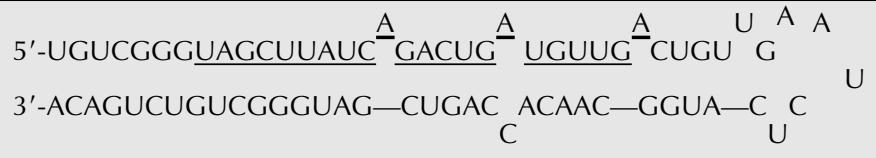 & + & + \\
\hline miR-21(U) & 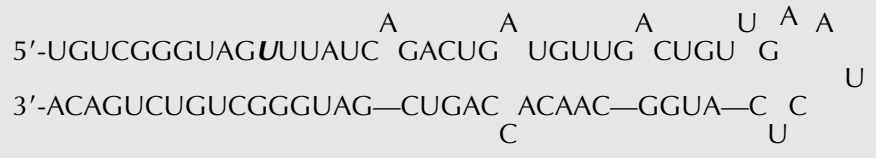 & + & - \\
\hline miR-21(C2) & 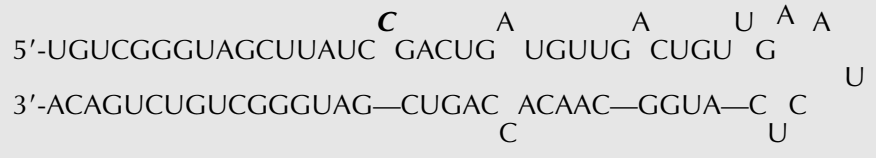 & + & + \\
\hline miR-21(C3) & $\begin{array}{l}\text { 5'-UGUCGGGUAGCUUAUC }{ }^{\mathrm{A}} \text { GACUG }{ }^{C} \text { UGUUG }^{\mathrm{A}} \mathrm{CUGU} \cup \mathrm{G}^{\mathrm{A}} \mathrm{A} \\
\text { 3'-ACAGUCUGUCGGGUAG-CUGAC ACAAC-GGUA-C }{ }_{C}^{\mathrm{C}}\end{array}$ & + & - \\
\hline miR-21(GGU) & 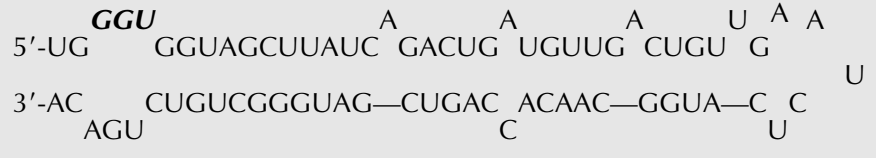 & - & - \\
\hline MiR-21(G1) & 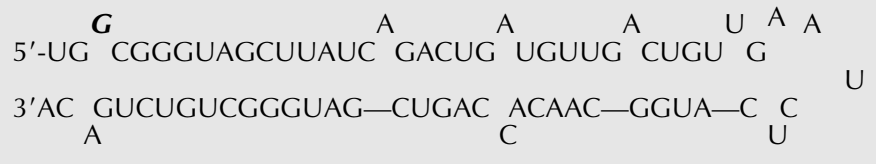 & \pm & \pm \\
\hline miR-21(G2) & 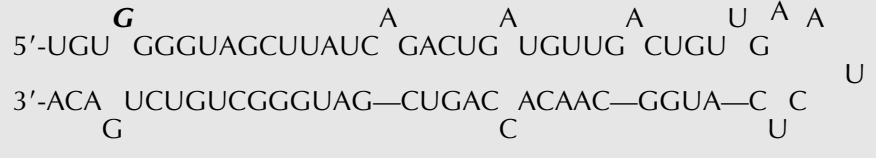 & + & + \\
\hline miR-21(U2) & 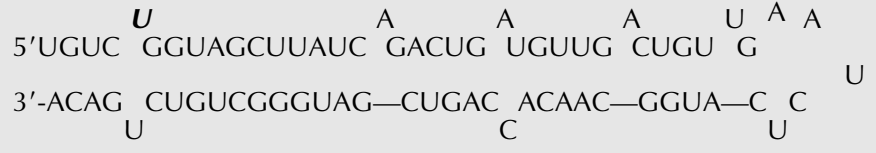 & + & + \\
\hline
\end{tabular}

The mature miR-21 sequence is underlined. See Table 1 legend for labeling.

expression of mRNAs bearing single, fully complementary target sites by inducing the degradation of the target mRNA. Using the same strategy, we constructed the pCMV/ miR-30-luc plasmid, which should express an siRNA targeted to the body of the firefly luciferase gene (Fig. 5A). The target sequence used was selected based on a published synthetic siRNA that has been used to inhibit luciferase expression in both the Drosophila and mammalian system (Elbashir et al. 2001a,b); therefore, we could compare the effects of in vitro synthesized siRNAs versus those transcribed in vivo. As shown in Figure 5B, lanes 2 and 4, the mature miR-30-luc miRNA/siRNA was detected in transfected cells. However, the same strategy did not work for miR-21-luc (Fig. 5B, lane 1). There is heterogeneity at the $5^{\prime}$ end of the miR-30-luc miRNA (Fig. 5B, cf. lanes 2 and 3), which was also observed with some other artificial siRNAs (data not shown). This finding likely relates to the as yet unknown rules governing the precise sites of cleavage in miRNA precursor molecules. The complement of miR-30luc was also made (data not shown). Importantly, the miR30-luc plasmid specifically reduced the activity of a cotransfected firefly luciferase expression plasmid in not only 293T cells (Fig. 5C) but also in HeLa cells and murine 3T3 cells (data not shown), a silencing effect very similar to that achieved by cotransfecting synthetic siRNA oligos (Elbashir et al. 2001a). As predicted, miR-30-luc effectively reduced the accumulation of luc mRNA (Fig. 4D,E, lane 5).

We introduced various point mutations into miR-30-luc (Fig. 5A) and analyzed RNA expression (Fig. 5B) and the effect on silencing (Fig. 5C). All of the miRNA mutants were made at similar levels (Fig. 5B, lanes 5-10). Changing the sequence in the strand identical to the mRNA did not affect RNAi [miR-21(C) in Fig. 5C]. Changing the sequences in the precursor strand predicted to anneal to the 
$\mathbf{A}$

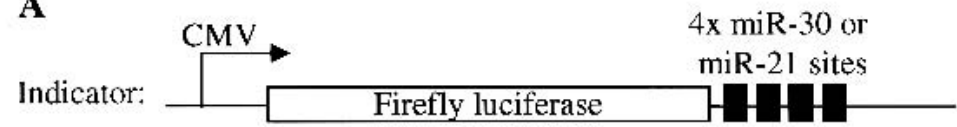

\section{B}
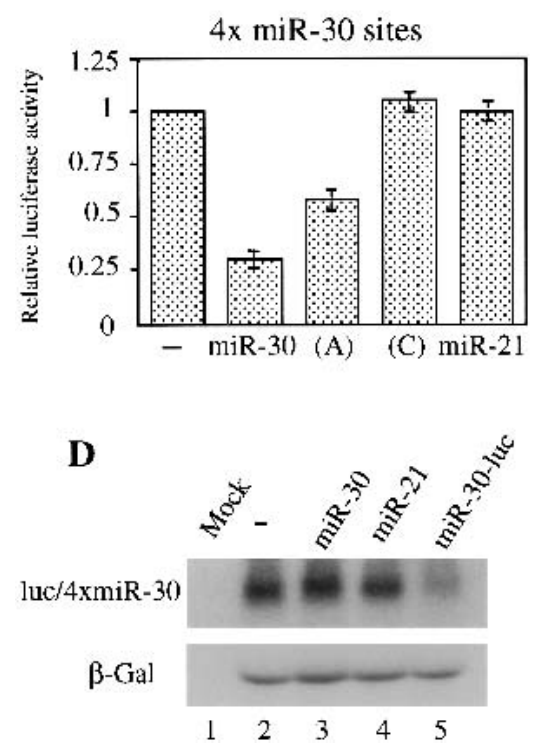

C

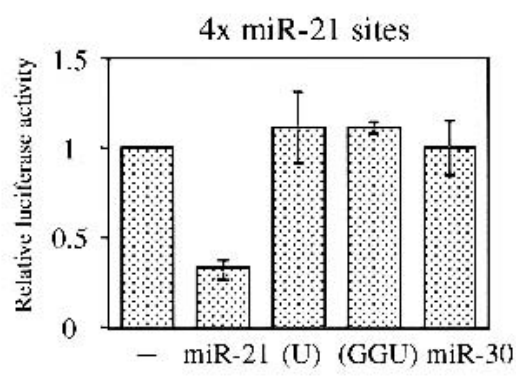

$\mathbf{E}$

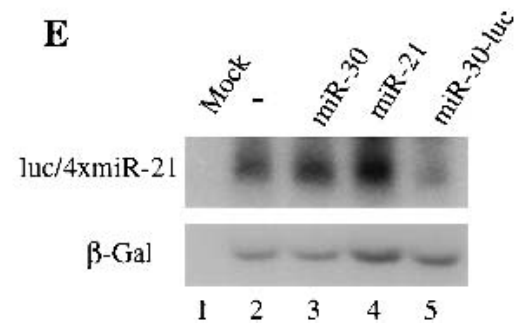

FIGURE 4. Inhibition of luciferase expression by miR-30 and miR-21. (A) Schematic of the luciferase indicator plasmids bearing four miR-30 or miR-21 target sequences (black boxes). (B) miR-30 inhibits the expression of the luciferase indicator bearing 4xmiR-30 target sites. A dual luciferase assay was performed, and the ratio of firefly luciferase to the internal control Renilla luciferase, from cells cotransfected with the parental $\mathrm{pBC} 12 / \mathrm{CMV}$ vector, was set at 1 (shown by -). (C) miR-21 inhibits the expression of the luciferase indicator encoding $4 x m i R-$ 21 target sites. Descriptions are the same as in $B$. $(D, E)$ Northern analyses detecting the luc indicator mRNA, or the $\beta$-gal internal control mRNA, in 293T cells cotransfected with the $\mathrm{pBC} 12 / \mathrm{CMV}$ control $(-)$ or the indicated miRNA expression plasmid.

target mRNA reduced, but did not eliminate, silencing activity (Fig. $5 \mathrm{C}$ ). The most disruptive mutations were three (A, G, U1) introduced near the center of the expected siRNA:mRNA helix, which nevertheless still repressed luciferase by $\sim 40 \%$.

\section{The miR-30 precursor is an unusually flexible miRNA expression vector}

In the course of studying miRNA expression, we found that mature miR-30 could apparently be expressed regardless of where its RNA stem-loop precursor was inserted within a longer transcript. The precursor could be located in an intron present in an HIV-1 transcript (Fig. 6A), or in a 3' UTR, and in both cases, mature miR-30 was produced (Fig. $6 \mathrm{~B}$, and data not shown). The full-length unspliced mRNA encoded by pgTat-miR-30 is retained in the nucleus unless the HIV-1 Rev nuclear mRNA export factor is also expressed (Malim et al. 1989; Zeng and Cullen 2002; data not shown). As demonstrated by a Northern analysis in Figure $6 \mathrm{~B}$, upon transfection of pgTat-miR-30, the $\sim 22$-nt mature miR-30 (indicated by an arrow) is detected in both the

nucleus and the cytoplasm of 293T cells, regardless of whether the Rev protein was present or not (Fig. 6B, lanes 2-5). In contrast, the $\sim 65$-nt pre-miRNA for miR-30 was exclusively nuclear in the absence of Rev (Fig. 6B, lanes 2,3), but also appeared in the cytoplasm upon Rev coexpression (Fig. 6B, lane 5). Therefore, processing of the longer, initial transcript to the $\sim 65$-nt miR-30 premiRNA can occur in the nucleus or in the cytoplasm. Although we could not detect endogenous mature miR-30 in 293 T cells (e.g., see Fig. 6B, lane 1), we were able to detect a very low level of the endogenous anti-miR-30 pre-miRNA in the nucleus (Fig. 6C, lane 1, indicated by an arrowhead). Therefore, these results imply that processing of endogenous miRNAs also initiates in the nucleus.

Genomic analysis has revealed that the stem-loop precursors encoding endogenous miRNAs can be found both as isolated copies and as tandem arrays (Lagos-Quintana et al. 2001; Lau et al. 2001). We therefore asked whether we would express more than one miRNA from a single initial RNA transcript. We tandemly cloned the precursor of miR30 and the precursor of an artificial miRNA, termed miR-30-E7, into the same cDNA under the control of RNA polymerase II-dependent CMV-IE promoter or the RNA polymerase III-dependent H1 promoter (Hannon et al. 1991). Upon transfection into 293T cells, both the CMV-IE and the H1-based construct expressed both the miR-30 and the miR-30-E7 miRNA and their complements (Fig. 7; not shown). Therefore, it is possible to express at least two, and possibly more, miR30-derived miRNAs by transcribing their precursors in tandem.

\section{DISCUSSION}

In this study, we have analyzed the effect of mutations in the miR-30 and miR-21 miRNA precursors on miRNA processing and function in transiently transfected human cells. For both human miR-30 and miR-21, base-pairing at the base of the precursor stem proved to be critical for mature miRNA production. We identified mutations in the miRNA precursors that prevent the mature miRNA from inhibiting indicator mRNA translation. We further demonstrated that single mismatches between a designed miRNA/siRNA and a target mRNA do not provide complete target discrimination. Finally, we present evidence suggesting that miR-30 
A

miR-30-1uc

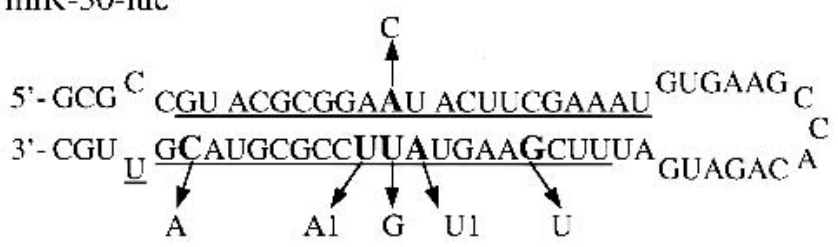

B

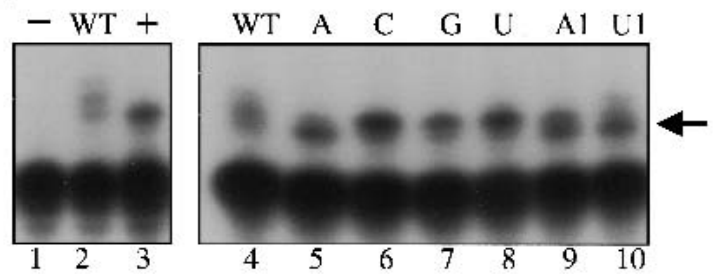

C

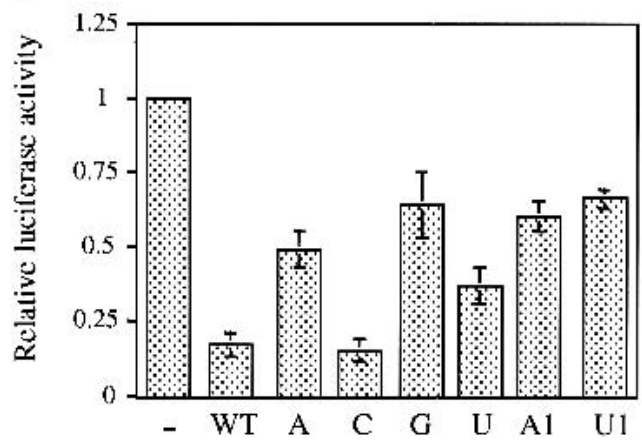

FIGURE 5. Mutational analysis of the miR-30-luc precursor. (A) Design of miR-30-luc. Sequences underlined are derived from the firefly luciferase mRNA, with the bottom strand being antisense to the mRNA. Single point mutations are indicated. (B) Expression of mature miR-30-luc variants as determined by primer extension. -, Cells transfected with pCMV/miR-21-luc (lane 1); +, $20 \mathrm{pg}$ of an oligonucleotide encoding the intended miR-30-luc, the underlined bottom strand in A (lane 3). Arrow indicates the expected position of miR30-luc. (C) Effect of miR-30-luc on luciferase expression. 293T cells were transfected with pCMV/luc, pRL-CMV, and plasmids encoding a wild-type or mutant miR-30-luc precursor. A dual luciferase assay was performed $2 \mathrm{~d}$ later. The ratio of firefly luciferase to Renilla luciferase observed in cells cotransfected with the parental $\mathrm{pBC} 12 / \mathrm{CMV}$ vector was set at 1 (shown by -).

production may involve two steps, that is, the initial excision of an $\sim 65$-nt pre-miRNA from a longer RNA transcript followed by cleavage, presumably by dicer, to produce the mature miRNA. At least the first of these two steps appears to occur primarily in the nucleus.

Until recently, little was known about how miRNAs are transcribed and processed except that the dicer ribonuclease is required to convert the precursor to the mature miRNA (Grishok et al. 2001; Hutvágner et al. 2001; Lee and Ambros 2001). We showed previously that miR-30 and anti-miR-30, encoded by the $3^{\prime}$ and $5^{\prime}$ arms of the miR-30 precursor, respectively, were expressed when cells were transfected with a CMV-IE based miRNA expression plasmid (Zeng et al. 2002). This result is somewhat unexpected, as normally one miRNA precursor gives rise to only one stable, mature miRNA, encoded by only one arm of the precursor (LagosQuintana et al. 2001; Lau et al. 2001; Lee and Ambros 2001). However, miR-30 appears unusual in this regard as endogenous miR-30 and anti-miR-30 (also termed miR-97) have both been detected in nontransfected human and murine cells (Lagos-Quintana et al. 2002; Mourelatos et al. 2002). Here, in the same system, the $\sim 65-n t$ miR-21 precursor produced mature miR-21, but not its complement. This suggests that our system, while artificial and simple, is a reliable one to express and study miRNAs in general, and it also strengthens our previous conclusion that, at least for some miRNAs, the precursor contains all the cis elements necessary to direct miRNA maturation. One possible explanation for the inability of three other predicted miRNA precursors (lin-4, let-7, and miR-27) to yield significant levels of mature miRNAs in our system, is that interactions between the miRNA precursor and flanking sequences may affect the architecture of the precursor RNA hairpin. This could conceivably add a level of regulation to miRNA expression in its natural context. Alternatively, excision of

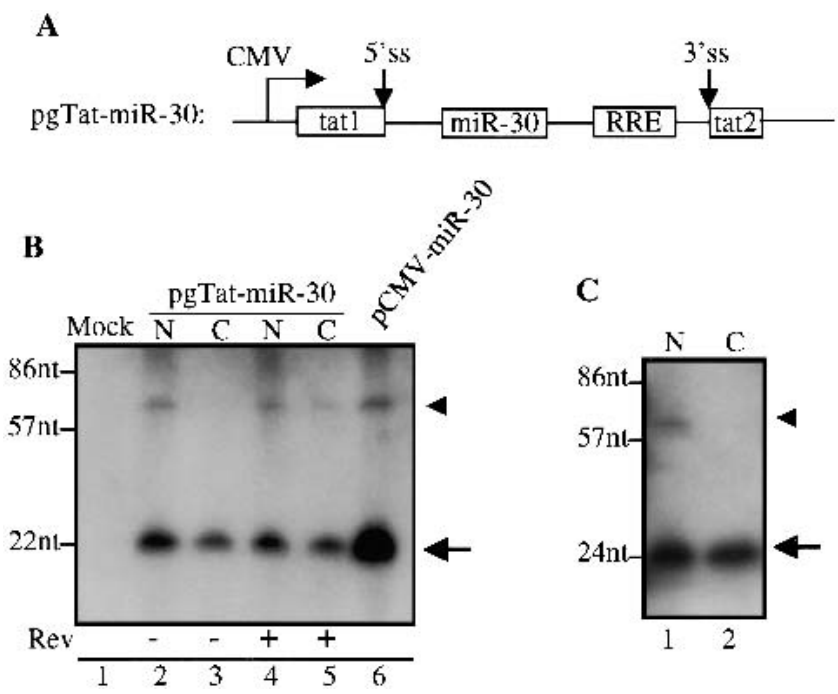

FIGURE 6. Subcellular localization of miRNAs. (A) Schematic of pgTat-miR-30. (B) Northern blotting for miR-30. Positions of DNA markers are shown in the left. The arrow marks the position of the mature miRNA and the arrowhead indicates the $\sim 65$-nt pre-miRNA. Lane 1, RNA from mock transfected cells; lane 2, nuclear RNA from cells transfected with pgTat-miR-30; lane 3, cytoplasmic RNA from cells transfected with pgTat-miR-30; lane 4, nuclear RNA from cells transfected with pgTat-miR-30 and pcRev; lane 5, cytoplasmic RNA from cells transfected with pgTat-miR-30 and pcRev; and lane 6, total RNA from cells transfected with pCMV-miR-30. (C) Northern blot containing nuclear RNA (N) and cytoplasmic RNA (C) from nontransfected $293 \mathrm{~T}$ cells was probed with an anti-miR-30-specific oligonucleotide. Labeling is the same as in $B$. Although detection of the endogenous anti-miR-30 required a much longer exposure than did detection of the exogenously expressed miR-30, it remains unclear why we were consistently unable to detect an endogenous miR-30 precursor signal in lane 1 of $B$. 


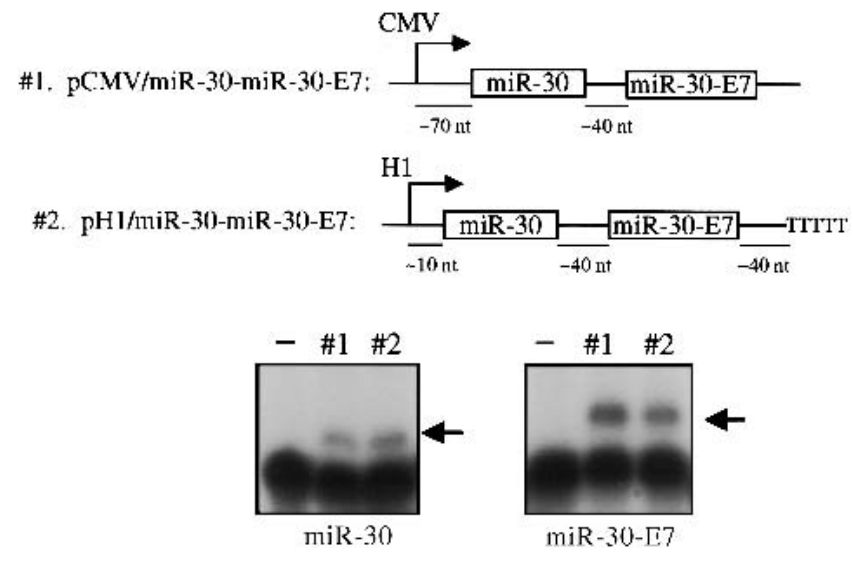

FIGURE 7. Two miRNAs can be expressed from a single transcript. Schematic of the CMV-IE-based pCMV/miR-30-miR-30-E7 and H1 promoter-based $\mathrm{pH} 1 / \mathrm{miR}-30-\mathrm{miR}-30-\mathrm{E} 7$ miRNA expression plasmids. Transfected $293 \mathrm{~T}$ cells produced both mature miR-30 and miR30-E7, as detected by primer extension (shown by arrows). -, RNA from mock transfected cells.

some miRNAs may require native flanking sequences longer than present in their reported, putative precursors (LagosQuintana et al. 2001).

Recently, several groups reported that artificial RNA hairpins, synthesized in vitro or expressed from Pol III promoters in vivo, are active in RNAi and are converted to $\sim 22$-nt siRNAs in mammalian cells (Brummelkamp et al. 2002; Paddison et al. 2002; Paul et al. 2002; Sui et al. 2002; Yu et al. 2002). We also showed that novel miRNA/siRNA can be made by substituting the stem sequences within the miR-30 precursor (Zeng et al. 2002). Thus, the stem sequence per se is not important for miRNA/siRNA processing as long as a certain level of base-pairing is maintained. As for the size of the artificial hairpin loop, predicted terminal loops ranging from 3 to $23 \mathrm{nt}$ have been successfully used. Therefore, the mutagenesis data presented here (Tables 1 and 2) are consistent with these recent reports, which suggest that the sequence/structure requirements for miRNA/siRNA processing are quite lax. Then how can a cell tell a bona fide miRNA precursor from just any RNA hairpin of $>50 \mathrm{nt}$ or so? It is, of course, entirely possible that some endogenous RNA hairpins are accidentally processed into miRNAs, yet at very low levels. In this paper we identify features in the precursors that facilitate miRNA maturation. Shortening the terminal loop to $4 \mathrm{nt}$, or creating a large bulge in the middle of the stem, significantly reduced miRNA expression. The biggest effect for both miR-30 and miR-21, however, resulted from disruption of the base-pairing at the base of the predicted precursor stem, outside the stem portion encoding the mature miRNAs (Tables 1 and 2). Taken together, there seems to be a minimal size requirement, from the bottom of the stem to the top of the loop, for miRNA processing and hence, presumably, for dicer recognition. Such a criterion may filter out many irrelevant RNA hairpins in vivo.

\section{Subcellular localization of miRNA processing}

After submission of this manuscript, Lee et al. (2002) reported that miRNA processing occurs in two steps. The initial endogenous miR-30 miRNA transcript was found to be a longer $(\geq 171 \mathrm{nt}) \mathrm{RNA}$, which appears comparable to the initial RNA transcript encoded by the pCMV-miR-30 plasmid. This "pri-miRNA" was processed in the cell nucleus to give rise to an $\sim 65$-nt pre-miRNA that was proposed to be identical to the apical region of the longer stem-loop structure that contains the mature miRNA sequence (e.g., Table 1). This pre-miRNA was then exported to the cytoplasm and cleaved, presumably by dicer, to give rise to the mature $\sim 22-n t$ miRNA (Lee et al. 2002). This hypothesis is consistent with earlier evidence suggesting that dicer is confined to the cytoplasm (Billy et al. 2001).

In this paper, we have observed that insertion of the miR-30 precursor into an intron, which is unable to exit the nucleus, still permits the production of readily detectable levels of miR-30 (Fig. 6), thus also arguing that the initial step in miRNA processing can occur in the nucleus. Using Northern analysis, we observed the production of not only mature miR-30 from both pCMV-miR-30 (Fig. 1) and pgTat-miR-30 (Fig. 6), but also of an $\sim 65$-nt RNA that, based on RPA, coincides with the apical $\sim 65 \mathrm{nt}$ of the proposed mir-30 precursor RNA stem-loop structure (Table 1) and that is identical in size to the mir-30 pre-miRNA reported by Lee et al. (2002). Our data, therefore, largely agree with the proposal of Lee et al. (2002) that miRNA processing normally occurs in two steps. A nuclear processing event that leads to the excision of the $\sim 65-n t$ pre-miRNA intermediate followed by the cytoplasmic excision of the mature miRNA by dicer. We note, however, that in our hands the $\sim 65$-nt miR-30 pre-miRNA was also detectable in the cell cytoplasm, but only when expressed as part of an mRNA that was being actively exported by the HIV-1 Rev protein (Fig. 6B, lane 5). This result clearly implies that excision of the pre-miRNA for miR-30 can also occur in the cytoplasm.

\section{Inhibition of gene expression using artificial miRNAs/siRNAs}

We have extended our previous observation (Zeng et al. 2002) that miR-30 can inhibit the translation of a CAT mRNA bearing four partially mismatched miR-30 target sites at its $3^{\prime}$ UTR, without significantly affecting mRNA expression levels. In this study, miR-21 also inhibited the expression of a CAT mRNA with multiple artificial miR-21 target sites, and both miR-30 and miR-21 could specifically inhibit the expression of a second indicator, luciferase mRNA, containing equivalent miRNA target sites. We have identified positions in miR-30 that are important for this inhibition; analogous positions in miR-21 are equally critical for this function (Figs. 2 and 3). Therefore, the ability of 
miRNAs to bind to mRNAs and inhibit gene expression appears to be a general phenomenon. The next step will be to study the mechanism of translation inhibition and to search for authentic miRNA targets.

The ability to express siRNAs from plasmids will undoubtedly have a significant impact on basic research, and potentially also on gene therapy and antiviral therapy. Therefore, the specificity of target recognition is an important issue. We have shown that single nucleotide mismatches between a $\sim 22-n t$ siRNA expressed from a plasmid, and a single, otherwise fully complementary target site present on an mRNA, lead to partial inhibition (Fig. 5). For example, a single mismatch at or near the center of complementarity still permitted luciferase activity to be inhibited by $\sim 40 \%$, as compared to $\sim 83 \%$ for a perfect match (Fig. 5). Such a mismatch tolerance has also been reported using synthetic siRNAs targeted to the endogenous human tissue factor mRNA (Holen et al. 2002). In contrast, in in vitro Drosophila extracts, artificial siRNAs with central point mutations were found to be completely inactive against a luciferase reporter mRNA (Elbashir et al. 2001b). There are two obvious explanations for this discrepancy. One is that complete base-pairing between the siRNA and the target is, in fact, not necessary for RNAi in human cells. The other is that the residual inhibition reflects translation inhibition by the 22-nt siRNA acting, in this case, as a canonical miRNA, like lin-4 (Olsen and Ambros 1999). The observed target specificity of these designed siRNAs has a number of possible implications for the use of siRNAs in experimental or clinical settings. For example, an siRNA designed to target a deleterious gene bearing a single point mutation may also affect the wild-type version, albeit with lower efficiency. On the other hand, if the target is a viral gene, then the siRNA may still be partially effective against viral variants bearing slightly different target sequences.

Most reported plasmid-based constructs express siRNAs from a Pol III promoter (Brummelkamp et al. 2002; Paddison et al. 2002; Yu et al. 2002). Pol III promoters are generally very strong promoters, but sequences surrounding the intended hairpin structure may affect siRNA production. Artificial miRNA expression plasmids based on the human miR-30 precursor appear to only need transcription, as they work with both Pol II and Pol III (Figs. 6 and 7). These precursors are also highly portable and multiple mir30-based miRNAs/siRNAs can apparently be excised independently from almost any location within a long transcript (Figs. 6 and 7; data not shown). There are some natural miRNAs that exist in clusters (miR-30 is not known to be one of them) and some of these miRNA precursors may exhibit the same flexibility as the miR-30 precursor (Lagos-Quintana et al. 2001; Lau et al. 2001). Once the detailed mechanism of miRNA processing is worked out, one could potentially construct a miRNA expression cassette that is devoid of any natural miRNA sequence, while maintaining portability and flexibility, that is optimized to express an exact siRNA sequences at the desired level from a suitable regulatable or tissue-specific promoter.

\section{MATERIALS AND METHODS}

\section{Molecular clones}

The expression plasmids pcRev, pDM128/RRE/4xmiR-30, pDM128/RRE, pBC12/CMV/ $\beta$-gal, pgTat, and pCMV-miR-30, as well as the parental expression plasmid $\mathrm{pBC12/CMV}$, have been previously described (Malim et al. 1989; Bogerd et al. 1995; Zeng et al. 2002). To construct pCMV-miR-21, the two DNA oligonucleotides, 5' -CGCTCGAGGATCCTGTCAGACAGCCCATCGA CTGGTGTTGCCATGAGATTCAACAG-3' and 5' -ACTCGAGAT CTGTCGGGTAGCTTATCAGACTGATGTTGACTGTTGAATCT CATGG-3', were annealed, extended by PCR, cleaved with XhoI, and used to replace the miR-30 sequence in pCMV-miR-30. To make pCMV/miR-30-luc, the two oligonucleotides, 5'-TACTC GAGATCTGCGCCGTACGCGGAATACTTCGAAATGTGAAGC CACAGATGA-3' and 5'-CGCTCGAGGATCCGCAACGTACGCG GAATACTTCGAAATCATCTGTGGCTTCACA-3', were annealed, extended by PCR, cut with $\mathrm{XhoI}$, and cloned as described above. To make pCMV/miR-21-luc, the two oligonucleotides, 5'-TACT CGAGATCTTGTCGGCTTTCGAAGTATTCCGCGTACGTCTGT

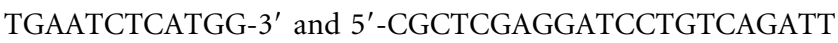
TCGAAGTATTCCGCGTACGGCCATGAGATTCAACAG-3', were used. To make pCMV/miR-30-E7, two oligonucleotides, 5' -TACT CGAGATCTGCGCACATTTACCAGCCCGACGCGTTGTGAAG CCACAGATGA-3' and 5' -CGCTCGAGGATCCGCAAACATTTA CCAGCCCGACGAGTTCATCTGTGGCTTCACA-3', were used and cloned as described above. Mutants of miR-30, miR-21, and miR-30-luc were constructed by PCR amplification using mutagenic primers and were verified by sequencing. pDM128/RRE/ 4xmiR-21, the indicator plasmid containing four copies of a miR21 target site, was constructed by cloning four tandem copies of miR-21 target sequence (Fig. 2B) into the XhoI site of pDM128/ RRE as previously described (Zeng et al. 2002). pCMV/luc was made by cloning the firefly luciferase gene between the NcoI and XhoI sites of pBC12/CMV. 4xmiR-30 and 4xmiR-21 DNA targets were then inserted into the XhoI site of $\mathrm{pCMV} / \mathrm{luc}$ to make the respective pCMV/luc indicators. To make pgTat-miR-30, PCRannealed oligonucleotides encoding the full-length precursor of miR-30 were digested with $B g l \mathrm{II}$ and $B a m \mathrm{HI}$ and cloned into the BglII site of pgTat. To make pCMV/miR-30-miR-30-E7, the PCRamplified miR-30 precursor sequence was digested with BglII and $B a m \mathrm{HI}$, treated with Klenow DNA polymerase, and cloned into the HpaI site of pCMV/miR-30-E7. pH1/miR-30-miR-30-E7 was constructed by ligating an NcoI-BglII DNA fragment containing miR-30 and the BglII and XhoI fragment containing miR-30-E7, both from $\mathrm{pCMV} / \mathrm{miR}-30$-miR-30-E7, into NcoI and XhoI sites present in a pGEM3Zf-derived plasmid containing the RNA polymerase III-dependent H1 promoter element (Hannon et al. 1991).

\section{Cell culture and transfection}

293T cells were transfected in 24-well tissue culture plates as previously described (Zeng et al. 2002). Each well received 0.2-0.4 $\mu \mathrm{g}$ of miRNA expression plasmid, $6 \mathrm{ng}$ of the $\mathrm{pBC} 12 / \mathrm{CMV} / \beta$-gal 
internal control plasmid, and 2.5-10 ng of the indicator plasmid. For dual-luciferase assays, $\sim 1 \mathrm{ng}$ of pRL-CMV (Promega) was included with the pCMV/luc plasmid, and $2 \mathrm{~d}$ later, luciferase activities were determined using the Dual-Luciferase Reporter Assay System (Promega). CAT and $\beta$-gal assays were performed as described (Bogerd et al. 1995). Data were averaged from at least three independent transfections with standard deviations indicated.

\section{RNA analysis}

Subcellular fractionation, RNA extraction, and Northern analysis were performed according to Zeng et al. (2002). The oligonucleotide used to detect miR-30 has the sequence: $5^{\prime}$-GCAGCTGCAAA CATCCGACTGAAAGCCC-3'; the oligonucleotide used to detect anti-miR-30: 5' -AGCTTCCAGTCGAGGATGTTTAC-3' . A 500bp fragment in the luciferase $5^{\prime}$ coding sequence was used to make a random-primed probe to detect luc mRNA by Northern blotting. The internal control, $\beta$-gal mRNA was detected using a $5^{\prime}$ $\sim 260$-bp probe fragment. For primer extension experiments, oligonucleotides labeled at the $5^{\prime}$ end with $\left[\gamma^{32} \mathrm{P}\right] \mathrm{ATP}$ and T4 polynucleotide kinase (Promega) were annealed to RNA at $37^{\circ} \mathrm{C}$ for 20 min, cooled at room temperature for $10 \mathrm{~min}$, and then extended at $42^{\circ} \mathrm{C}$ for $\sim 15$ min using AMV reverse transcriptase (Promega). Products were analyzed by electropheresis on an $8 \mathrm{M}$ urea/15\% polyacrylamide gel. The oligonucleotides used in primer extension assays were as follows: for miR-30 detection, 5' -TATGCAGCTG CAAACATCCGAC-3'; for miR-21, 5'-GCTAGTCAACATCAGT CTGATA-3'; for anti-miR-21, 5'-GCTGTCAGACAGCCCATCG ACT-3'; for miR-30-luc, 5'-GAGCAACGTACGCGGAATACTT3'; and for miR-30-E7, 5'-GTACACAACTCGTCGGGCTGGT-3'.

\section{ACKNOWLEDGMENTS}

The publication costs of this article were defrayed in part by payment of page charges. This article must therefore be hereby marked "advertisement" in accordance with 18 USC section 1734 solely to indicate this fact.

Received June 10, 2002; accepted September 26, 2002.

\section{REFERENCES}

Banerjee, D. and Slack, F. 2002. Control of developmental timing by small temporal RNAs: A paradigm for RNA-mediated regulation of gene expression. BioEssays 24: 119-129.

Bernstein, E., Caudy, A.A., Hammond, S.M., and Hannon, G.J. 2001. Role for bidentate ribonuclease in the initiation step of RNA interference. Nature 409: 363-366.

Billy, E., Brondani, V., Zhang, H., Muller, U., and Filipowic, W. 2001. Specific interference with gene expression induced by long, double-stranded RNA in mouse embryonal teratocarcinoma cell lines. Proc. Natl. Acad. Sci. 98: 14428-14433.

Bogerd, H.P., Fridell, R.A., Madore, S., and Cullen, B.R. 1995. Identification of a novel cellular cofactor for the Rev/Rex class of retroviral regulatory proteins. Cell 82: 485-494.

Brummelkamp, T.R., Bernards, R., and Agami, R. 2002. A system for stable expression of short interfering RNAs in mammalian cells. Science 296: 550-553.
Elbashir, S.M., Harborth, J., Lendeckel, W., Yalcin, A., Weber, K., and Tuschl, T. 2001a. Duplexes of 21-nucleotide RNAs mediate RNA interference in cultured mammalian cells. Nature 411: 494-498.

Elbashir, S.M., Martinez, J., Patkaniowska, A., Lendeckel, W., and Tuschl, T. 2001b. Functional anatomy of siRNAs for mediating efficient RNAi in Drosophila melanogaster embryo lysate. EMBO J. 20: 6877-6888.

Grishok, A., Pasquinelli, A.E., Conte, D., Li, N., Parrish, S., Ha, I., Baillie, D.L., Fire, A., Ruvkun, G., and Mello, C.C. 2001. Genes and mechanisms related to RNA interference regulate expression of the small temporal RNAs that control C. elegans developmental timing. Cell 106: 23-34.

Hamilton, A.J. and Baulcombe, D.C. 1999. A species of small antisense RNA in posttranscriptional gene silencing in plants. Science 286 950-952.

Hammond, S.M., Bernstein, E., Beach, D., and Hannon, G.J. 2000. An RNA-directed nuclease mediates post-transcriptional gene silencing in Drosophila cells. Nature 404: 293-295.

Hannon, G.J., Chubb, A., Maroney, P.A., Hannon, G., Altman, S., and Nilsen, T.W. 1991. Multiple cis-acting elements are required for RNA polymerase III transcription of the gene encoding H1 RNA, the RNA component of human RNase P. J. Biol. Chem. 266: 22796-22799.

Holen, T., Amarzguioui, M., Wiiger, M.T., Babaie, E., and Prydz, H. 2002. Positional effects of short interfering RNAs targeting the human coagulation trigger tissue factor. Nucleic Acids Res. 30: $1757-1766$.

Hutvágner, G. and Zamore, P.D. 2002. RNAi: Nature abhors a doublestrand. Curr. Opin. Gen. Dev. 12 225-232.

Hutvágner, G., McLachlan, J., Pasquinelli, A.E., Bálint, É., Tuschl, T., and Zamore, P.D. 2001. A cellular function for the RNA-interference enzyme dicer in the maturation of the let-7 small temporal RNA. Science 293: 834-838.

Knight, S.W. and Bass, B.L. 2001. A role for the RNase III enzyme DCR-1 in RNA interference and germ line development in Caenorhabditis elegans. Science 293: 2269-2271.

Lagos-Quintana, M., Rauhut, R., Lendeckel, W., and Tuschl, T. 2001. Identification of novel genes coding for small expressed RNAs. Science 294: 853-858.

Lagos-Quintana, M., Rauhut, R., Yalcin, A., Meyer, J., Lendeckel, W., and Tuschl, T. 2002. Identification of tissue-specific microRNAs from mouse. Curr. Biol. 12: 735-739.

Lau, N.C., Lim, L.P., Weinstein, E.G., and Bartel, D.P. 2001. An abundant class of tiney RNAs with probable regulatory roles in Caenorhabditis elegans. Science 294: 858-862.

Lee, R.C. and Ambros, V. 2001. An extensive class of small RNAs in Caenorhabditis elegans. Science 94 862-864.

Lee, R.C., Feinbaum, R.L., and Ambros, V. 1993. The C. elegans heterochronic gene lin-4 encodes small RNAs with antisense complementarity to lin-14. Cell 75: 843-854.

Lee, Y., Jeon, K., Lee, J.-T., Kim, S., and Kim, V.N. 2002. MicroRNA maturation: stepwise processing and subcellular localization. EMBO J. 21: 4663-4670.

Malim, M.H., Hauber, J., Le, S.-Y., Maizel, J.V., and Cullen, B.R. 1989. The HIV-1 rev trans-activator acts through a structured target sequence to activate nuclear export of unspliced viral mRNA. $\mathrm{Na}$ ture 338: 254-257.

Mourelatos, Z., Dostie, J., Paushkin, S., Sharma, A., Charroux, B., Abel, L., Rappsilber, J., Mann, M., and Dreyfuss, G. 2002. miRNPs: A novel class of ribonucleoproteins containing numerous microRNAs. Genes \& Dev. 16: 720-728.

Olsen, P.H. and Ambros, V. 1999. The lin-4 regulatory RNA controls developmental timing in Caenorhabditis elegans by blocking LIN14 protein synthesis after the initiation of translation. Dev. Biol. 216: 671-680.

Paddison, P.J., Caudy, A.A., Bernstein, E., Hannonm G.J., and Conklin, D.S. 2002. Short hairpin RNAs (shRNAs) induce sequencespecific silencing in mammalian cells. Genes \& Dev. 16: 948-958.

Paul, C.P., Good, P.D., Winer, I., and Engelke, D.R. 2002. Effective 
expression of small interfering RNA in human cells. Nature Biotech. 20: 505-508.

Reinhart, B.J., Slack, F.J., Basson, M., Pasquinelli, A.E., Bettinger, J.C., Rougvie, A.E., Horvitz, H.R., and Ruvkun, G. 2000. The 21nucleotide let-7 RNA regulates developmental timing in Caenorhabditis elegans. Nature 403: 901-906.

Sui, G., Soohoo, C., Affar, E.B., Gay, F., Shi, Y., Forrester, W.C., and Shi, Y. 2002. A DNA vector-based RNAi technology to suppress gene expression in mammalian cells. Proc. Natl. Acad. Sci. 99: 5515-5520.

Yu, J.-Y., DeRuiter, S.L., and Turner, D.L. 2002. RNA interference by expression of short-interfering RNAs and hairpin RNAs in mammalin cells. Proc. Natl. Acad. Sci. 99 6047-6052.

Zamore, P.D., Tuschl, T., Sharp, P.A., and Bartel, D.P. 2000. RNAi: Double-stranded RNA directs the ATP-dependent cleavage of mRNA at 21 to 23 nucleotide intervals. Cell 101: 25-33.

Zeng, Y. and Cullen, B.R. 2002. RNA interference in human cells is restricted to the cytoplasm. RNA 8: 855-860.

Zeng, Y., Wagner, E.J., and Cullen, B.R. 2002. Both natural and designed micro RNAs can inhibit the expression of cognate mRNAs when expressed in human cells. Mol. Cell. 9: 1327-1333. 

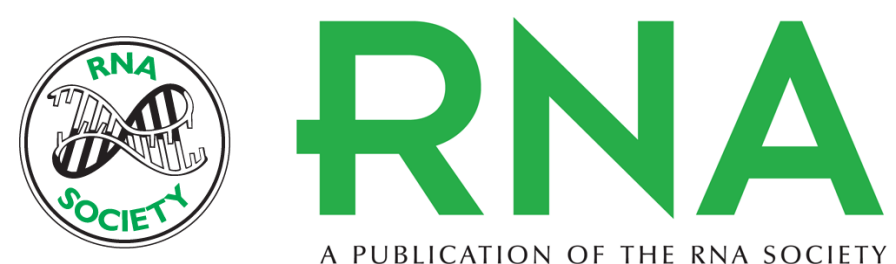

A PUBLICATION OF THE RNA SOCIETY

\section{Sequence requirements for micro RNA processing and function in human cells}

YAN ZENG and BRYAN R. CULLEN

RNA 2003 9: 112-123

References This article cites 32 articles, 15 of which can be accessed free at: http://rnajournal.cshlp.org/content/9/1/112.full.html\#ref-list-1

\section{License}

Email Alerting Receive free email alerts when new articles cite this article - sign up in the box at the Service top right corner of the article or click here. 